\title{
Intermittency, quasiperiodicity and chaos in probe-induced ferroelectric domain switching
}

\author{
A. V. levlev 1,2, S. Jesse ${ }^{2}$, A. N. Morozovska ${ }^{3}$, E. Strelcov² , E. A. Eliseev ${ }^{4}$, Y. V. Pershin ${ }^{5}$, A. Kumar ${ }^{2,6}$, \\ V. Ya. Shur ${ }^{1 \star}$ and S. V. Kalinin ${ }^{2 \star}$
}

Memristive materials and devices, which enable information storage and processing on one and the same physical platform, offer an alternative to conventional von Neumann computation architectures. Their continuous spectra of states with intricate field-history dependence give rise to complex dynamics, the spatial aspect of which has not been studied in detail yet. Here, we demonstrate that ferroelectric domain switching induced by a scanning probe microscopy tip exhibits rich pattern dynamics, including intermittency, quasiperiodicity and chaos. These effects are due to the interplay between tip-induced polarization switching and screening charge dynamics, and can be mapped onto the logistic map. Our findings may have implications for ferroelectric storage, nanostructure fabrication and transistor-less logic.

$\mathrm{C}$ omplex time dynamics including periodic, quasiperiodic and chaotic behaviours ${ }^{1,2}$ have long been recognized as a hallmark of strongly non-equilibrium systems ranging from convection and climate to reactions on catalytic surfaces ${ }^{3-5}$. The chaotic time behaviour necessitates the existence of fractal strange attractors in the phase space of the system, establishing an intrinsic relationship between dynamics and spatial complexity. Significant efforts have been devoted towards spatial visualization of these behaviours, as well as the development of control algorithms through macroscopically measurable variables to evoke or suppress these behaviours ${ }^{6,7}$.

At the same time, non-equilibrium systems often exhibit spatial complexity, including the formation of intricate periodic and fractal-like patterns in diffusion-limited aggregation, reaction and solidification fronts, percolation systems $s^{3,4}$, and even one-dimensional (1D) droplet or bubble jets ${ }^{8}$. Despite multiple examples of spatiotemporal complexity in physics, biology and chemistry, only limited information is available on controlling these behaviours spatially, precluding their practical use. Furthermore, these behaviours were assumed to be irrelevant to systems with binary physical states, such as computer memories and data storage. Here we demonstrate that polarization switching in ferroelectric materials under the action of a biased scanning probe microscopy (SPM) tip can give rise to complex spatiotemporal dynamics, including intermittency, quasiperiodicity and chaos. This behaviour is ascribed to the balancing of polarization switching and screening charge-mediated depolarization fields, and opens exciting opportunities for the physical implementation of systems such as cellular automata.

As a model system, we have chosen the ferroelectric $\mathrm{LiNbO}_{3}$ surface, shown in Fig. 1. Application of a bias to the SPM tip in contact with the surface leads to local polarization reversal below the tip, thereby resulting in the formation of a switched domain. This switched domain can subsequently be detected in the piezoresponse force microscopy mode, visualizing domains on the basis of the magnitude and direction of the piezoelectric response ${ }^{9,10}$. Here, a set of bias pulses was applied, resulting in a clearly visible chain of domains of equal size. Note that domains are formed at each spatial point of bias application, and that the domain size is uniform, indicative of the high quality of the material. This behaviour was explored extensively in the context of ferroelectric data storage ${ }^{11,12}$ and fundamental studies of domain formation and stability $^{13,14}$. Generally, domain growth follows kinetic laws with domain size linearly proportional to the tip bias (above a certain threshold corresponding to domain nucleation) and approximately logarithmic with the pulse time ${ }^{11}$, and is traditionally attributed to domain wall pinning in the disordered medium ${ }^{13}$.

However, unusual effects are observed once the spacing between the points of bias application is varied, as illustrated in Fig. 2. On decreasing the separation between the bias application locations, the domain sizes become non-equal, often with pronounced transient behaviour between the first and subsequent domains in the chain. For certain parameters, we observe intermittency, namely only every second domain is formed or domains of non-equal size with clear double periodicity are observed. On further decreasing the spacing, period tripling or the formation of non-periodic or longperiodic structures is observed. This behaviour is highly unusual for domain switching, and is clearly reminiscent of the period doubling and chaotic dynamics present in dynamic systems ${ }^{8}$, where the number of locations at which bias is applied along the surface plays the role of discrete time and the domain radius is the system state.

We explore the variation of point spacing and tip bias (for a fixed bias pulse duration of $250 \mathrm{~ms}$ ) as shown in Fig. 2a-i to construct the diagram shown in Fig. 2j. Application of biases below the nucleation threshold does not lead to domain formation (region I). Large biases result in continuous domain switching (domain size exceeds spacing between domains) and the formation of a 'stripe' domain (region II). Application of moderate biases for large separations

\footnotetext{
${ }^{1}$ Ferroelectric Laboratory, Institute of Natural Sciences, Ural Federal University, 620000 Ekaterinburg, Russia, ${ }^{2}$ The Center for Nanophase Materials Sciences, Oak Ridge National Laboratory, Oak Ridge, Tennessee 37922, USA, ${ }^{3}$ Institute of Physics, National Academy of Sciences of Ukraine, 46, pr. Nauki, 03028 Kiev, Ukraine, ${ }^{4}$ Institute for Problems of Materials Science, National Academy of Sciences of Ukraine, 3, Krijianovskogo, 03142 Kiev, Ukraine, ${ }^{5}$ Department of Physics and Astronomy, and the University of South Carolina Nanocenter, University of South Carolina, Columbia, South Carolina 29208, USA, ${ }^{6}$ Centre for Nanostructured Media, School of Mathematics and Physics, Queen's University Belfast, Belfast BT7 1NN, UK. *e-mail: shur@labfer.ru; sergei2@ornl.gov
} 
a

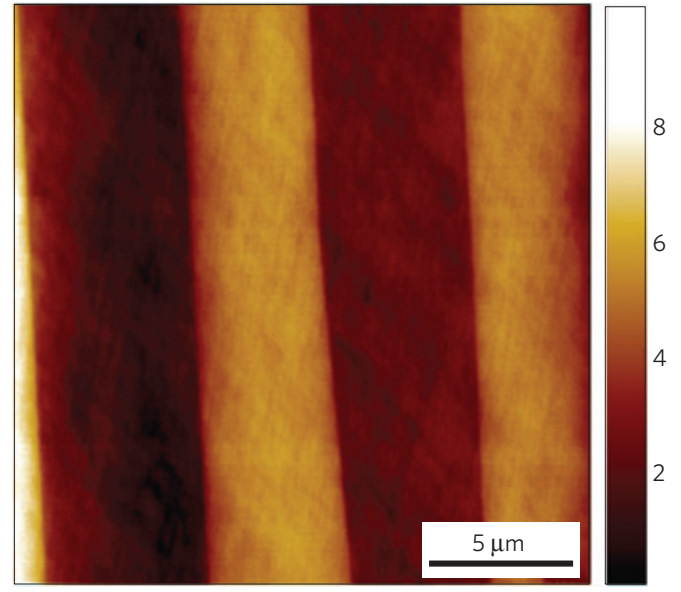

c

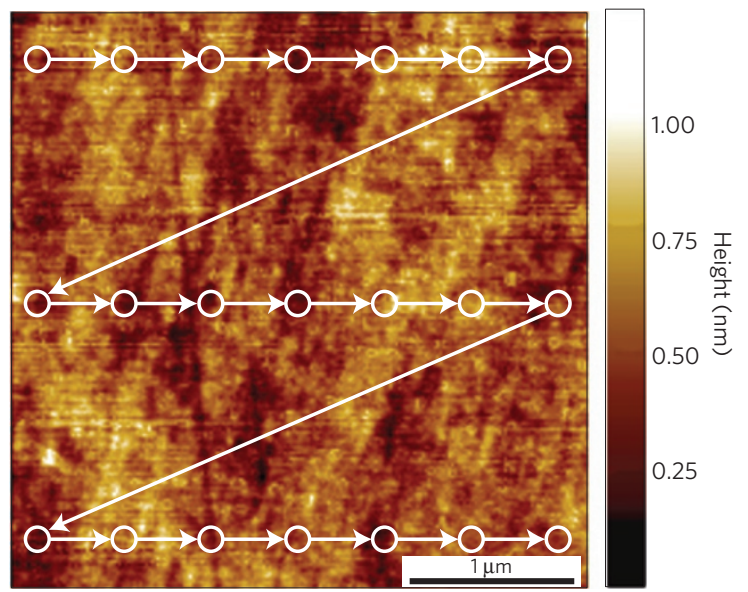

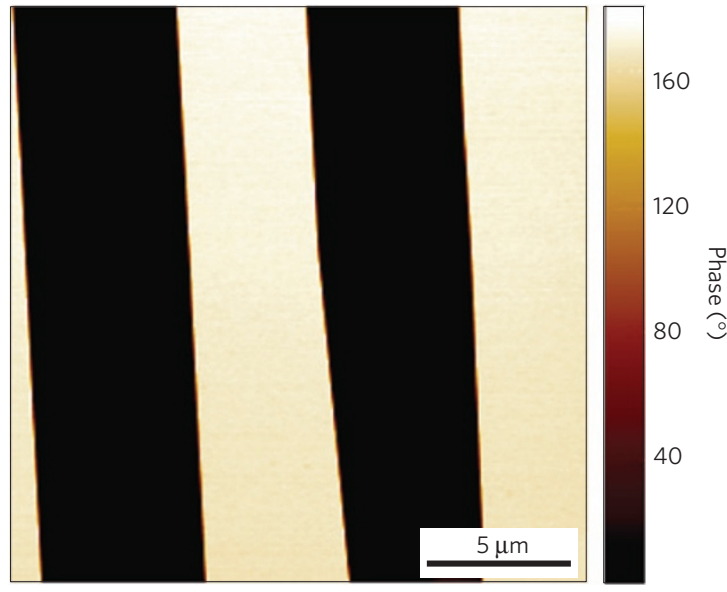

d

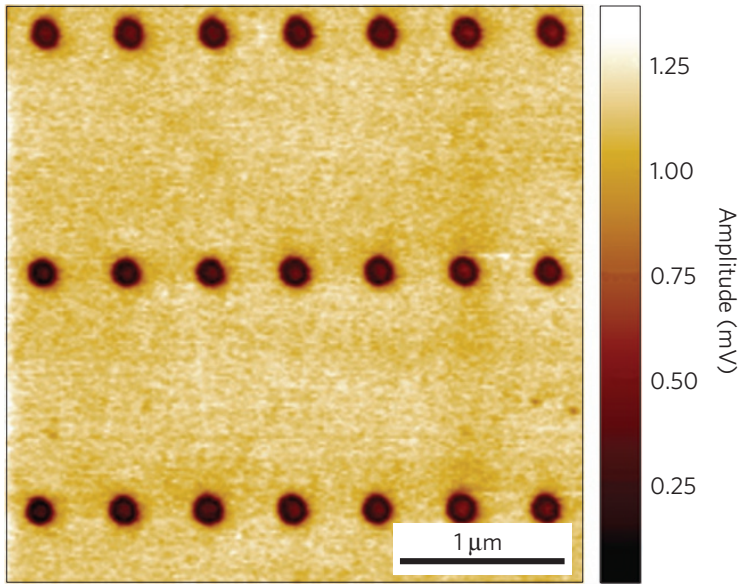

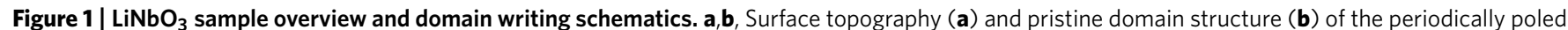
$\mathrm{LiNbO}_{3}$ surface. $\mathbf{c}$, Schematic of domain writing by a biased SPM probe within a single domain region. $\mathbf{d}$, Domain writing by negative bias in the regions with upward directions of the spontaneous polarization yields a rectangular array of switched domains, with uniformly sized domains forming at each location where bias has been applied.

results in well-defined domain chains formed by uniform domains at each point of bias application, already explored earlier in the context of information storage ${ }^{11,15-17}$. For very large separations, this corresponds to non-interacting domains in region IIIa. The uniform chains can also form for weak domain interaction, as shown in region IIIb. The boundary between the two is established on the basis of the transient behaviour in the chain, namely whether the first domain is larger than subsequent ones. Finally, unexpected behaviour is observed in region IV, where intermittency and quasiperiodic switching is observed. Some of these behaviours are illustrated in Fig. 2, including uniform chain formation, period doubling, period tripling, quasiperiodic and chaotic behaviours.

These observations suggest that forming domains interact with each other, resulting in feedback-type mechanisms that under certain conditions can give rise to chaotic regimes. Here, we explore possible origins of such behaviour, analyse the domain-domain interaction, and explore whether these mechanisms can give rise to chaotic regimes. The primary factors that lead to domain interactions in ferroelectric materials include electrostatic fields and strain. For uniaxial materials such as lithium niobate, the strain effects (in the off-field state) are expected to be minimal, suggesting that the role of electrostatic interactions needs to be explored. The free energy of a ferroelectric domain nucleated under an SPM probe sensitively depends on the screening on the top surface and in fact polarization switching is impossible in the absence of efficient screening ${ }^{18,19}$. In ambient environment, the screening is performed by ionic species, probably hydroxyls, $\mathrm{OH}^{-}$, and protons, $\mathrm{H}^{+}$, that are readily available as a result of dissociative water adsorption ${ }^{20,21}$. A similar role can be fulfilled by polarization gradients in the near-surface regions (for example, due to partial exchange of $\mathrm{Li}^{+}$ by protons in $\mathrm{LiNbO}_{3}$ ) that give rise to effective space charge layers and long-range depolarization fields. The screening by near-surface band bending (ferroelectric semiconductors) is energetically less favourable (because the corresponding length scale is the Debye length in the material as opposed to the bond length) and is typically realized only under high-vacuum conditions ${ }^{22,23}$.

For ionic screening, polarization switching in the selected region results in the formation of excess uncompensated charge, and the process can be represented as:

$$
\left([+\mathrm{P}]-\mathrm{OH}^{-}\right)+\mathrm{H}_{2} \mathrm{O}+2 \mathrm{e}^{-} \rightarrow\left([-\mathrm{P}]-\mathrm{H}^{+}\right)+2 \mathrm{OH}^{-}
$$

Here, $\left([+\mathrm{P}]-\mathrm{OH}^{-}\right)$is the positive polarization charge bound with the screening hydroxyl group (see refs 24,25 for a discussion of equilibrium degree of screening), and $\left([-\mathrm{P}]-\mathrm{H}^{+}\right)$is the negative polarization charge bound with a screening proton. Note that maintaining local quasi-electroneutrality during polarization switching requires that the switching phenomena be coupled to surface electrochemical processes.

For the example above, formed hydroxyls diffuse laterally across the sample surface, creating a 'halo' surrounding the switched area. Note that this charge injection has repeatedly been observed by, for 
a

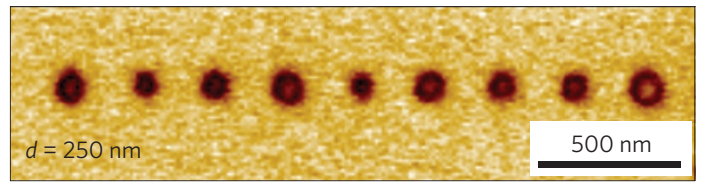

b

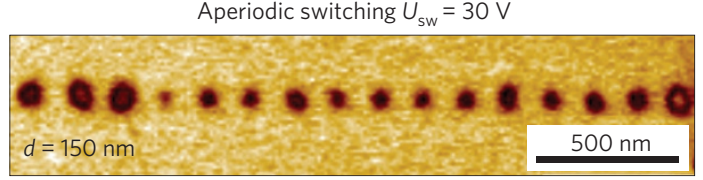

c

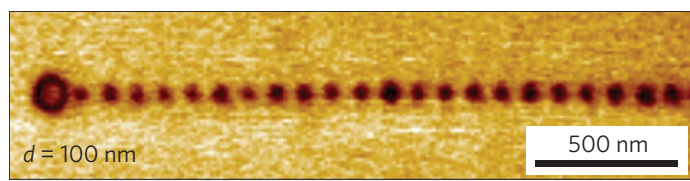

Period tripling $U_{s w}=30 \mathrm{~V}$

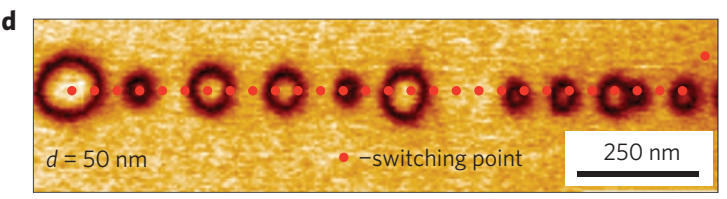

Intermittent switching $U_{s w}=40 \mathrm{~V}$
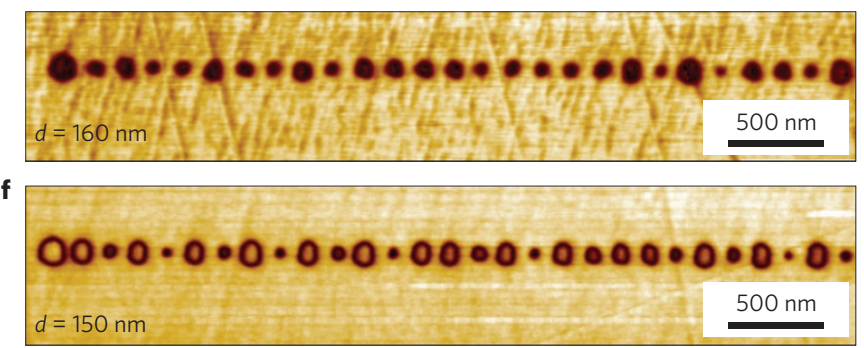

g

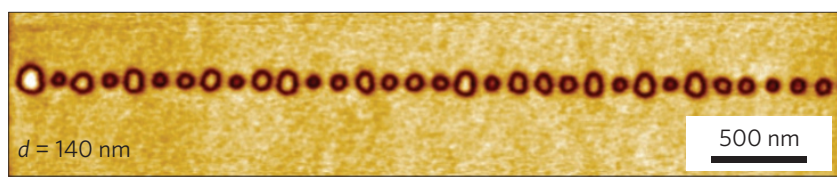

h

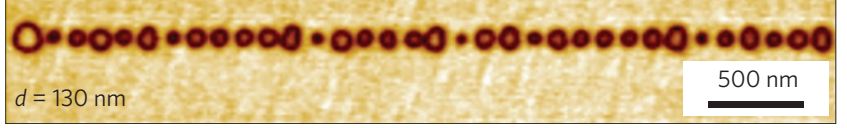

Domains merging $U_{\text {sw }}=50 \mathrm{~V}$

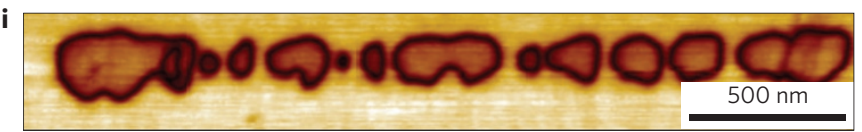

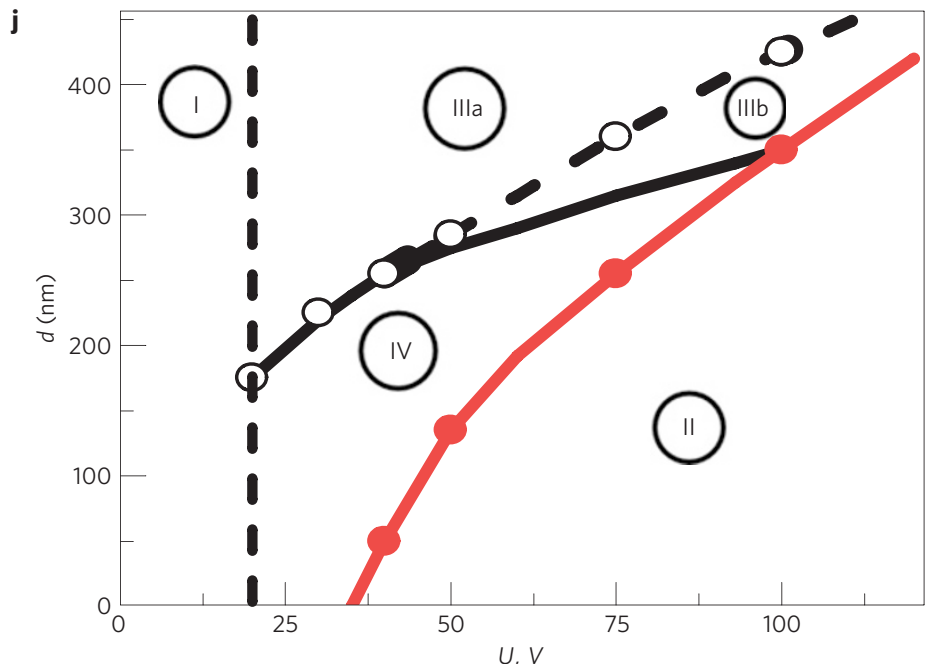

Figure 2 | Evolution of domain morphologies in a single chain as a function of writing conditions. a-d, Domains formed after switching by pulses with

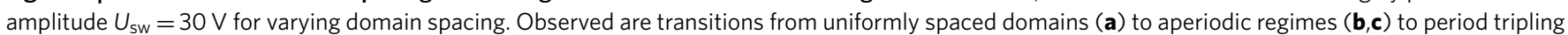
(d). e-h, Domain morphologies at $U_{\text {sw }}=40 \mathrm{~V}$. Note the clearly visible period doubling (f) and intermittent behaviours (g,h). $\mathbf{i}$, Domain merging at

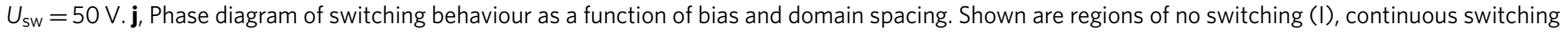
(II), isolated uniform domains (IIla), uniform domains with transient (IIIb), and quasiperiodic and chaotic behaviour (IV).

example, Kelvin probe force microscopy ${ }^{16,26,27}$. The spatial extent and lifetime of the 'halo' and the structure of the depolarization fields is controlled by the diffusional mobility of the surface ions and the thickness of the surface water layers. The depolarization fields, in turn, can affect domain nucleation behaviour in the adjacent locations (non-local Le Chatelier effect), giving rise to nontrivial proximity effects. This suggests that for ionic compensation, the variation of the state of the water layer should affect the character of switching in the chains (unlike bulk ionic screening or band bending effects).

To get insight in this behaviour, the measurements were performed as a function of temperature and relative humidity, as depicted in Fig. 3. On increasing the temperature for parameters corresponding to intermittent switching at room temperature, we observe a transition to the regular writing. Note the clearly visible transient behaviour, corresponding to region IIIb in Fig. 2j. A series of remarkable transitions is observed on increasing the humidity. For (nominally) zero humidity, the writing yields a series of equal-sized domains. For higher humidity, the onset of a period doubling is observed at $25 \%$ relative humidity. At higher humidity, quasiperiodic structures are observed for $35 \%$ and long-range periodicity (up to $8-10$ domains) is observed for $50 \%$ relative humidity. For $60 \%$ humidity the domains again become uniform. For a further increase of humidity, the domain size starts 


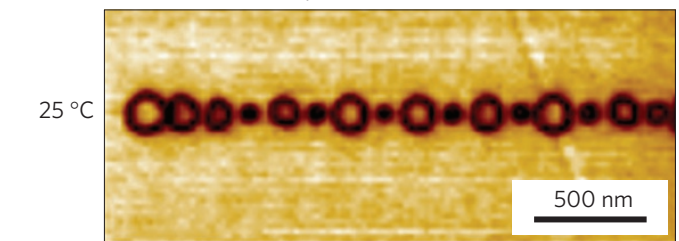

b

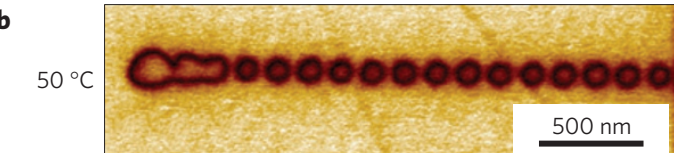

$75^{\circ} \mathrm{C}$

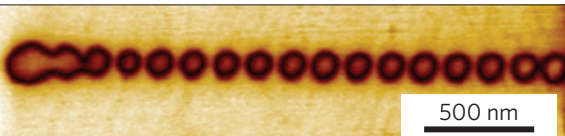

d

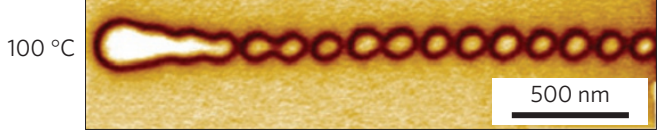

e

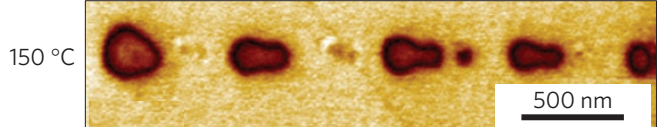

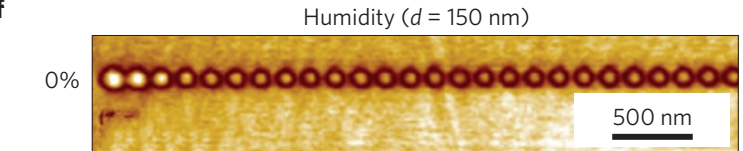

g

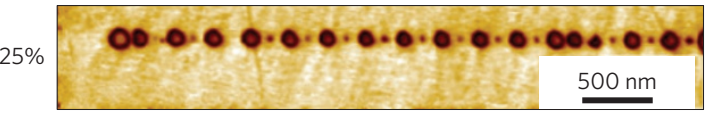

h

$40 \%$

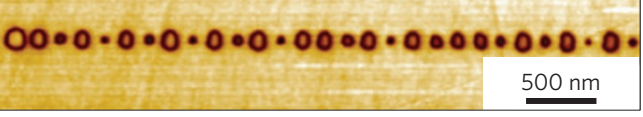

i

$50 \%$

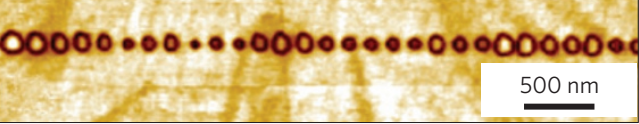

$60 \%$
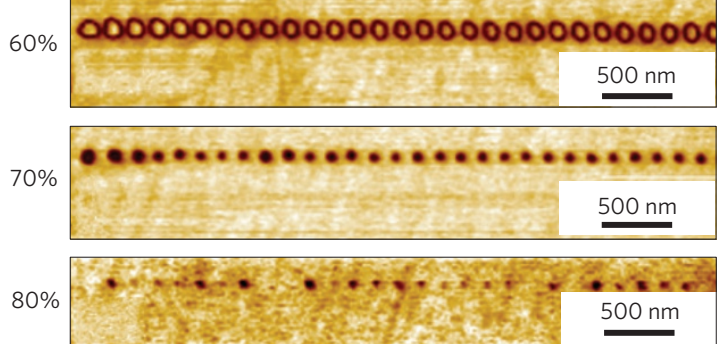

Figure 3 | Evolution of the domain patterns. a-e, Variable-temperature measurements. Note the rapid change of the switching character between 100 and $150^{\circ} \mathrm{C}$ from period doubling (a) to uniform chains (b-d) to long-range periodicity (e). $\mathbf{f - I}$, Evolution of the switching pattern as a function of relative humidity at room temperature. The data illustrate a transition between uniform switching (f), period doubling ( $\mathbf{g}, \mathbf{h})$, long-range periodicity (i) and back to uniform switching (j-I). Switching disappears at high humidity (decrease of domain sizes from $\mathbf{j}$ to $\mathbf{l}$; no domain formed at $90 \%$ ). Measurements in a-e are taken at $40 \%$ humidity (defined at room temperature); measurements $\mathbf{f}-\mathbf{I}$ are taken at room temperature.

to reduce, transient behaviour becomes less pronounced, and for (nominally) 90\% humidity and above, domains no longer form at this bias. This behaviour is also confirmed by locally measured hysteresis loops (not shown).

We rationalize these observations, on the basis of equation (1). Here, for low humidity the thickness of the adsorbed water layer on the surface is minimal, and hence the charge halo around the domain is highly localized and does not affect the formation of adjacent domains. For intermediate humidity values, the charges are localized in the vicinity of the formed domain, and intermittent dynamics are observed. Finally, for high humidity the charge spreads rapidly across the sample surface, obviating this effect. At the same time, the water layer effectively screens the surface, precluding polarization switching (for example, compare with piezoresponse force microscopy and switching in liquids ${ }^{28,29}$ ).

With the character of interaction between domains traced to the surface charge dynamic effect, we proceed further to quantify these interactions and map them onto classical models of dynamic systems. Here, we describe the system state at the $n$th location by the domain radius, $r_{n}$. In the initial location, $r_{0}=r(U)$, where $r(U)$ is the kinetic law for domain size versus voltage for non-interacting domains. In location 1 , formation of the domain in location 0 creates a non-zero screening charge density and associated depolarization fields that affect domain nucleation in subsequent location 2, and so on. This transient behaviour can be clearly seen in, for example, Fig. 3d, with initial domains always largest and subsequent transient behaviour over $3-5$ points. At some point, the initial conditions become irrelevant and the system adopts a behaviour described by a stationary solution for the iterative sequence $r_{n+1}=F\left(r_{n}\right)$.

Here, we consider the simplest model for domain interaction mediated by the long-range depolarization fields, as depicted in
Fig. 4a,b. The effect of the liquid layer with mobile ions is modelled through the presence of a surface dielectric layer of physical thickness $l$ and the effective Debye length of mobile ions $R_{\mathrm{d}}$. It can be shown that in this case the effective layer thickness is $H=l+R_{\mathrm{d}} \varepsilon_{\mathrm{g}} / \varepsilon_{\mathrm{f}}$, where $\varepsilon_{\mathrm{g}}$ and $\varepsilon_{\mathrm{f}}$ are the dielectric constants of the dielectric and screening layers respectively. For a (sufficiently thick) $\mathrm{H}_{2} \mathrm{O}$ layer on a $\mathrm{LiNbO}_{3}$ surface $\varepsilon_{\mathrm{g}} \approx \varepsilon_{\mathrm{f}}$ and $H \approx R_{\mathrm{d}}$. The domain interaction is determined by the depolarization field, $E_{\mathrm{d}}\left(r_{n}, d\right)$, induced by the pre-existent $n$th domain of radius $r_{n}$, and the strength of this interaction can be evaluated analytically for a prescribed surface charge density. The shape of the new domain $r_{n+1}$ can then be estimated from the condition

$$
E_{\mathrm{d}}\left(r_{n}, d\right)+E_{\text {tip }}\left(r_{n+1}, R_{0}\right)=E_{\text {cr }}
$$

equating the sum of tip field and depolarization field to the critical field for domain wall motion, $E_{\mathrm{cr}}$. The SPM tip field, $E_{\text {tip }}\left(r_{n+1}, R_{0}\right)$ is determined by the dielectric properties of the material and the effective tip radius of curvature $R_{0}$, and can be described using the sphere-plane $\operatorname{model}^{30-33}$. For a nearly isotropic material such as $\mathrm{LiNbO}_{3}$, parametric equation (2) can be solved to yield a relationship between domain radii of subsequent domains, $x_{n+1}=F\left(x_{n}\right)$, as

$$
x_{n+1}^{2} \approx\left(\frac{u}{1-g\left(\delta, x_{n}, h\right)}\right)^{2 / 3}-1
$$

Here $x_{n+1}=r_{n+1} / R_{0}$ and $x_{n}=r_{n} / R_{0}$ are the normalized domain radii and $u=U / E_{\mathrm{cr}} R_{0}$ is the normalized tip bias. The function $g\left(\delta, x_{n}, h\right)=E_{\mathrm{d}}\left(d, r_{n}, H\right) / E_{\mathrm{cr}}$ is the normalized depolarization field, $\delta=d / R_{0}$ is the normalized distance between centres of the domains, and $h=H / R_{0}$ is the parameter determined by the water layer on the 


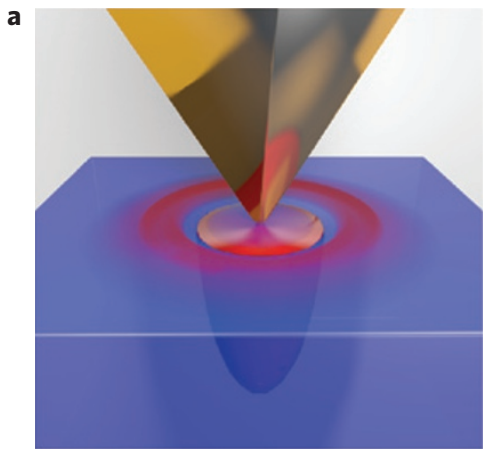

b
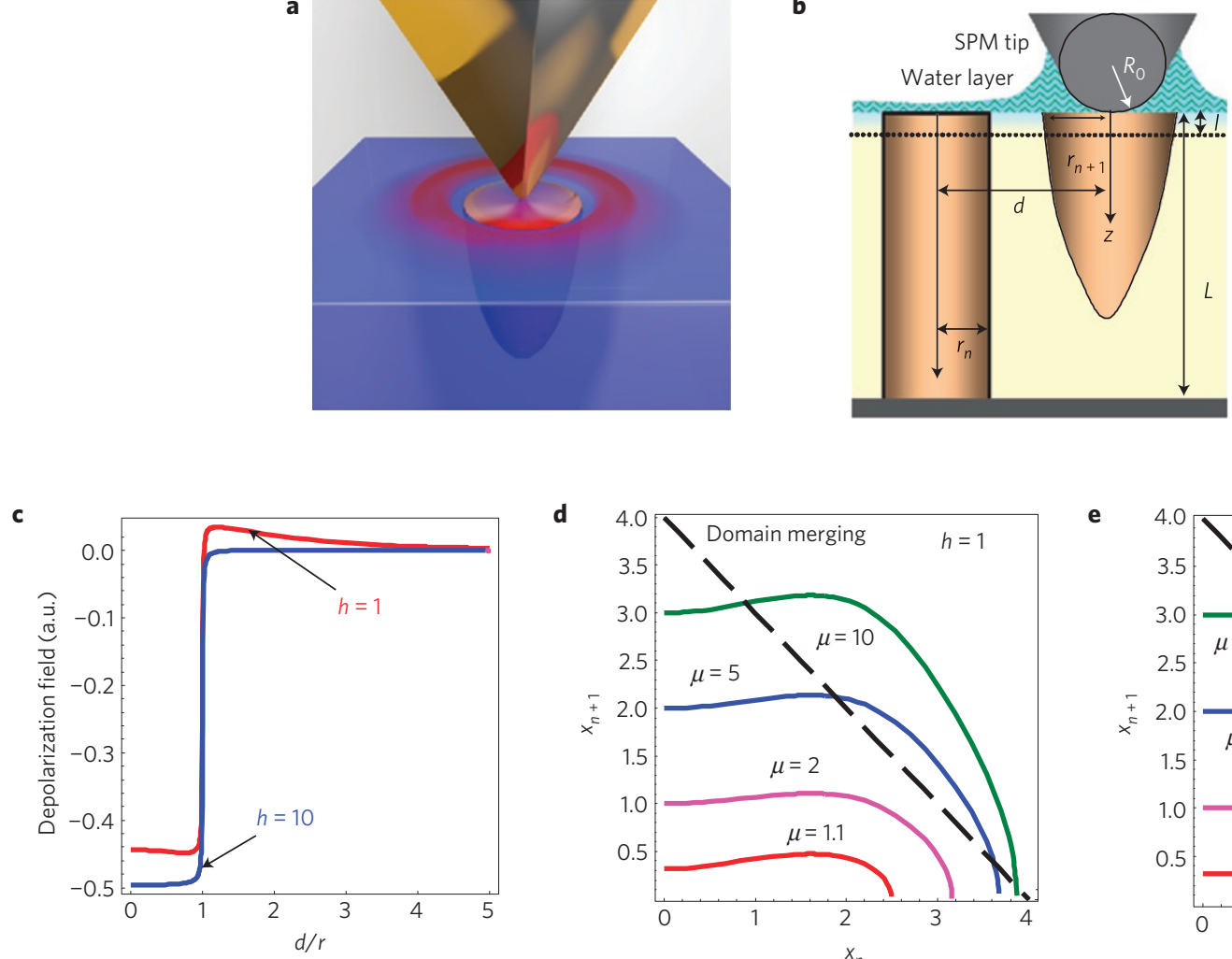

d

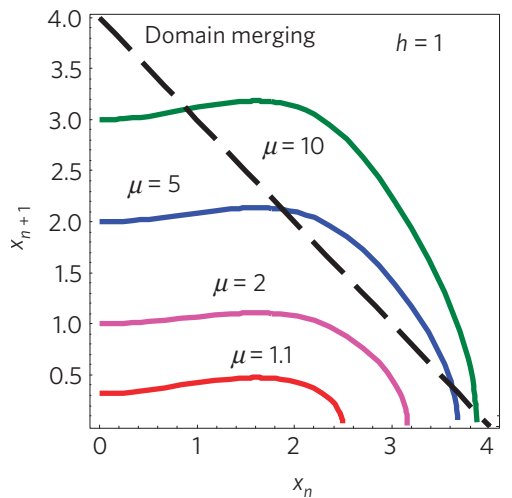

e

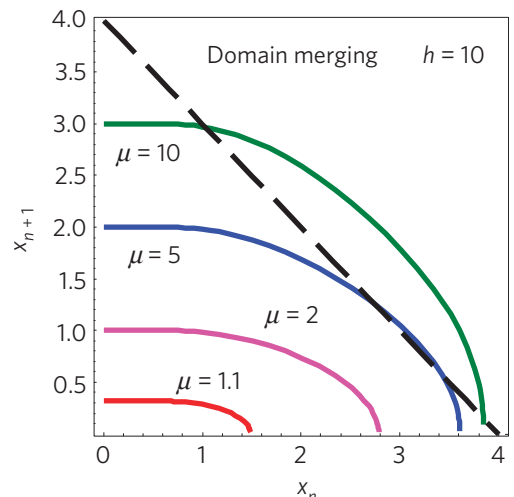

Figure 4 | Modelling domain writing. a, Domain with a ring of surface charge. $\mathbf{b}$, Schematic of the domain interaction calculations. c, Depolarization field distribution calculated from equation (2) (parameters of screening charge can be found in the Supplementary Information). $\mathbf{d}, \mathbf{e}$, Dependence $x_{n+1}\left(x_{n}\right)$ calculated using equation (3) for parameters $\delta=4, \lambda=10$, and humidity $h=10(\mathbf{d}), h=1$ (e), and different values of $\mu=u^{2 / 3}$ indicated near the curves.

surface (that is, humidity). The control parameters varied during the SPM experiment are the normalized bias $u$ and the distance between the centres of the domains $\delta$.

In the framework of equation (3), the nucleation of a single domain in the absence of the pre-existing domain is possible for $u>1$ and the bias dependence of domain shape is $x_{n+1}=\sqrt{u^{2 / 3}-1}$, in agreement with Landauer-Molotskii theory ${ }^{34}$. This behaviour is affected by the domain-domain interactions, and the calculated $x_{n+1}=F\left(x_{n}\right)$ values for different $h$ values are shown in Fig. 4 d,e. The non-monotonic behaviour of $x_{n+1}\left(x_{n}\right)$ is present at moderate humidity $h=1$, but absent at higher or lower values. Thus, calculated dependences can be numerically analysed and bifurcation diagrams can be constructed as shown in Fig. 5a,b (see also Supplementary Information), demonstrating the emergence of a rich spectrum of dynamic behaviours for certain values of humidity.

To establish the universality class of observed behaviours, we perform a Taylor series expansion of the depolarization field. From the symmetry of the problem, the series expansion can be constructed in terms of a single variable $(h / \delta) x_{n}^{2}$, where $\delta$ is the distance between the domains, as: $g \approx \lambda \chi(h / \delta) x_{n}^{2}\left(1-\beta x_{n}^{2}\right)$. Here $\lambda=P_{\mathrm{S}} / \varepsilon_{0} \varepsilon E_{\mathrm{cr}}$ is a parameter determined by the spontaneous polarization and critical field for the wall motion; $\chi$ and $\beta$ are expansion coefficients determined by the depolarization field (see Supplementary Information). Subsequently rescaling $z_{n}=(\alpha / w) \beta x_{n}^{2}+$ $(1 / 2)-(\alpha / 2 w)$, where $\alpha=1+\sqrt{(w-1)^{2}+4 w \beta\left(u^{2 / 3}-1\right)}$ and $w=2 u^{2 / 3}(\lambda \chi h / 3 \delta)$, yields the well-known logistic map $z_{n+1}=$ $\alpha z_{n}\left(1-z_{n}\right)$. Here $\alpha$ is a single master parameter determined by material and tip properties (Supplementary Table 1). Properties of the logistic map and the corresponding bifurcation diagram are well studied ${ }^{2}$, notably including the onset of a period doubling bifurcation at $\alpha=3$, quadrupling at $1+\sqrt{6}$, and subsequent opening of a period doubling cascade. At $\alpha$ approximately equal to 3.57 , there is an onset of chaotic behaviour, with isolated ranges of $\alpha$ that show non-chaotic stability windows.

Avoiding simplifications involved in the determination of the universality class, the domain chain behaviour can be analysed in the parameter space corresponding to the experimentally controlled variables. The phase diagram of switching behaviour as a function of dimensionless voltage $u$ and domain spacing $\delta$ is shown in Fig. 5c. Corresponding domain patterns are illustrated in Fig. 5d. Note the clearly visible regions of no domain formation, uniform domains, and period doubling and chaotic regimes, reminiscent of experimental data. This analysis can be taken one step further by matching the regions in Figs $2 j$ and $5 c$. Using a probe size of $R_{0}=30 \mathrm{~nm}$ as estimated from the ferroelectric wall width, this scaling allows the critical field to be estimated as $E_{\mathrm{cr}}=0.67 \mathrm{~V} \mathrm{~nm}^{-1}$ from the theoretical condition $u_{\mathrm{cr}}=\left(U_{\mathrm{cr}} / E_{\mathrm{cr}} R_{0}\right)=1$ (Fig. $5 \mathrm{c}$ ) and the experimental value of the critical voltage $U_{\mathrm{cr}}=20 \mathrm{~V}$ (Fig. 2j). The value of the critical field is well within the margins of a Peierls field calculated as $0.4-0.8 \mathrm{~V} \mathrm{~nm}^{-1}$ using the Peierls barrier of the domain wall motion $W_{\mathrm{p}}$ of value $125 \mathrm{~mJ} \mathrm{~m}^{-2}$ calculated by density functional theory ${ }^{35}$ and $225 \mathrm{~mJ} \mathrm{~m}^{-2}$ from an empirical study (we used $W_{\mathrm{p}} \propto P_{\mathrm{S}} E_{\mathrm{cr}} a_{0}$, spontaneous polarization $P_{\mathrm{S}}=0.75 \mathrm{C} \mathrm{m}^{-2}$, wall width $a_{0}=0.4 \mathrm{~nm}$ ). Using the parameter $h=2$, the effective water layer thickness was found to be $H=h R_{0}=60 \mathrm{~nm}$. Note that this analysis suggests that the only primary parameter affecting the onset of complex dynamic regimes is the effective water layer thickness, which is controlled by humidity.

Note that the theoretical behaviour explored above is very sensitive to the details of the screening process. Although the general picture involving the formation of the ring of excess surface 


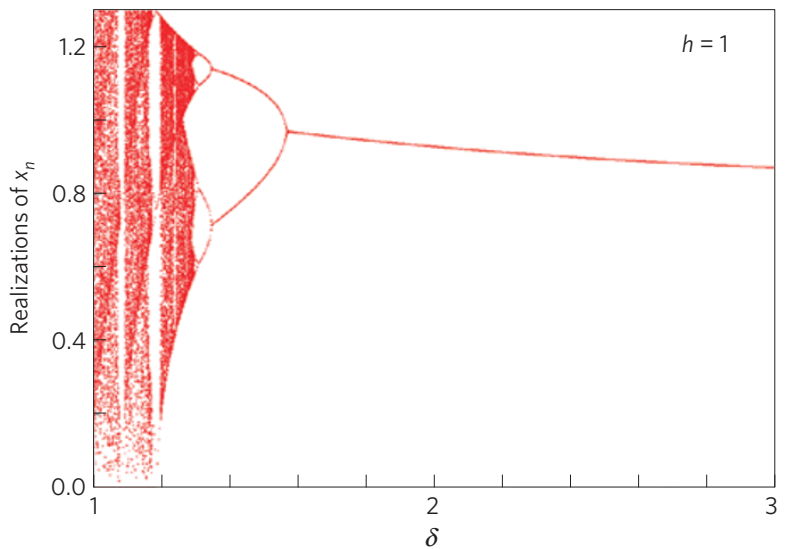

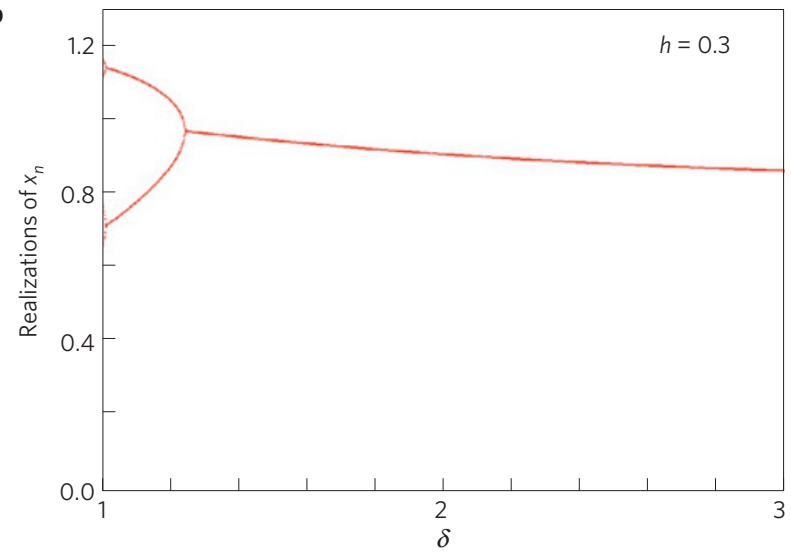

c

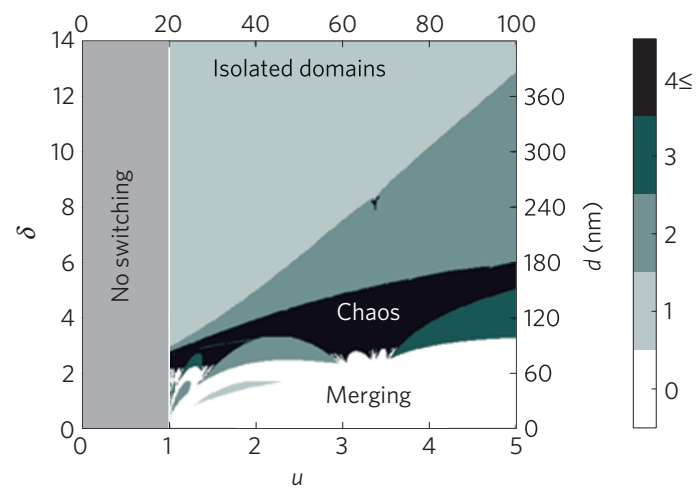

Uniform chain $(\delta=2.0)$

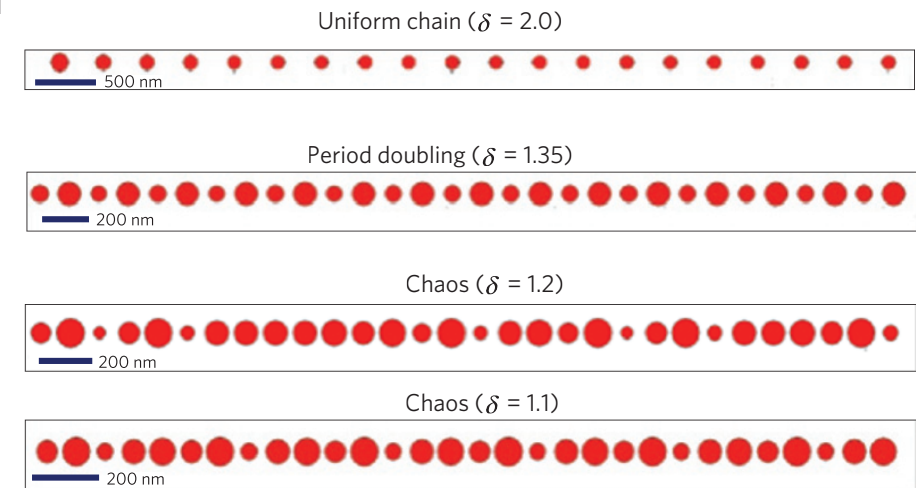

Figure 5 | Numerical solutions for various conditions. a,b, Bifurcation maps obtained by direct numerical solution of equation (3) for parameter $h=0.3$ (a) and 1 (b), and bias $u=1.15$. c, Phase diagram of switching behaviour as a function of dimensionless voltage $u=\left(U / E_{\mathrm{cr}} R_{0}\right)$ and domain spacing $\delta=d / R_{0}$ calculated for $h=1$. The colour bar indicates the number of bifurcation solutions. Note the qualitative similarity between the experimental diagram in Fig. $2 \mathrm{j}$ and the theoretical diagram (c). Also shown are axes obtained by rescaling to experimentally measurable parameters for $R_{0}=30 \mathrm{~nm}$ and $E_{\mathrm{cr}} R_{0}=20 \mathrm{~V}$. Using bulk materials parameters $P_{\mathrm{S}}=0.75 \mathrm{C} \mathrm{m}^{-2}, \varepsilon_{33}=30$ and $\varepsilon_{11}=84$, the critical field for wall motion can be estimated as $E_{\mathrm{cr}}=0.67 \mathrm{Vmm} \mathrm{nm}^{-1}$ and the effective width of the water layer is $H=60 \mathrm{~nm}$. d, Several realizations of domain sequences obtained by numerical solution of equation (3). The size of the red circles corresponds to the domain size. The parameters are chosen as shown in $\mathbf{b}$. Note the agreement between calculated patterns and those observed experimentally in Fig. 2.

charge that suppresses nucleation in the vicinity of domains can be expected to be correct, the observation of chaotic behaviour requires the function $x_{n+1}=q\left(x_{n}\right)$ to be non-monotonic; that is, interaction between domains at short ranges should be repulsive but at long ranges attractive. This behaviour is visible in Fig. 4d,e. To explore this behaviour directly, Fig. 6 shows the experimental data of $x_{n+1}=q\left(x_{n}\right)$. For large domains (or small separations) the pre-existent domains clearly suppress the formation of domains at $n+1$ locations. For small $r_{n}$ (large separations or small domain sizes), there is a small upward trend, clearly visible on the regularized curve.

We further note that the consequence of an increasing value, $\mathrm{d} q / \mathrm{d} x_{n}>0$ for $x_{n} \geq 0$, would be that the presence of a domain facilitates nucleation at large separations. To establish this effect, we perform writing with high biases as illustrated in Fig. 6b,c. Application of a bias in primary locations along the chain induces the formation of secondary domains where no bias is applied. This suggests that at large distances nucleation is indeed increased, in agreement with the upward trend of the curve in Fig. 6a for small $x_{n}$.

To summarize, we report the surprising observations of intermittency, quasiperiodicity and chaotic dynamics during SPM tip-induced polarization switching on ferroelectric surfaces. These experiments unambiguously indicate that polarization switching involves a coupling between switching and electrochemical processes, and the electrochemical step can be a rate-limiting element and also result in long-range interactions.

From the point of view of potential applications, the domain interaction effect demonstrated in this paper is very interesting in connection to the emergent computing paradigm known as memcomputing ${ }^{36}$. Here, massively parallel dynamics of networks of memristors or memcapacitors ${ }^{37}$ could allow a significant improvement in information processing capabilities, provide a solution to the von Neumann bottleneck problem, and result in future brain-like computing architectures ${ }^{36}$. Clearly, the main requirement for memcomputing - the ability to store and process information on the same physical platform-is met in our system. The domain radius is a natural quantity to encode binary or analogue information depending on a specific task. In fact, the experimental results presented in Fig. 2 already demonstrate the possibility of binary logic operations. For this purpose, it is convenient to use the period doubling regime (Fig. $2 \mathrm{f}$ ), when the domains of small (logic 0$)$ and large (logic 1) radii alternate. In terms of logic operations, the domain alternation is the NOT gate. By using the interaction of three domains, the logic NOR (functionally complete) and AND gates can be realized (see Supplementary Information for more details). This shows that our system in principle possesses a universal computing ability. 
a

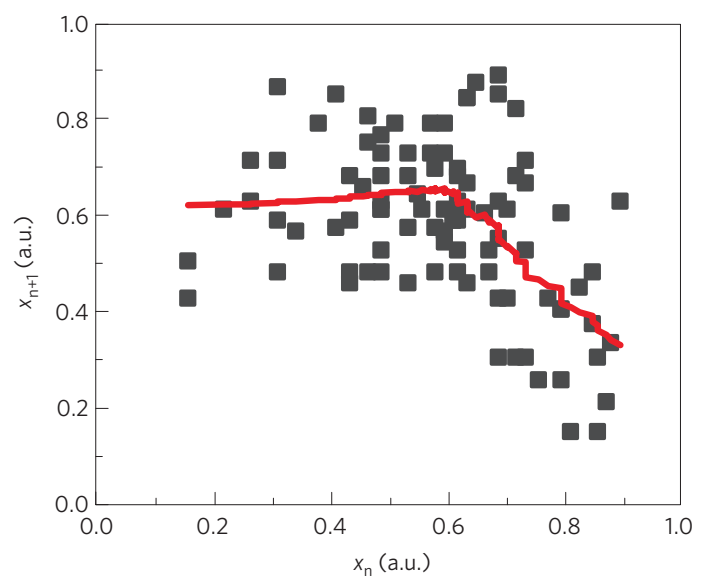

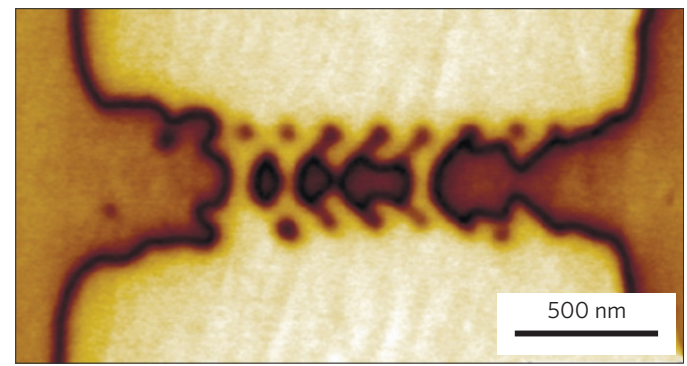

c

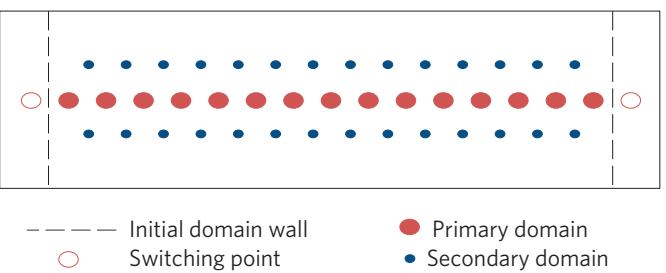

Figure 6 | Comparison of experimental and modelled results. a, Experimentally determined recursive relation $x_{n+1}=q\left(x_{n}\right)$ and smoothened dependence (red line). Suppression of domain nucleation in the proximity of pre-existent domains is clearly seen $\left(x_{n}>0.5\right)$, as well as a small upward trend for $x_{n}<0.4$ (compare to Fig. 4e). b, Nucleation of the secondary domains for writing of the domain chains across narrow stripe domain $(U=100 \mathrm{~V}, d=150 \mathrm{~nm})$, illustrating long-range domain-induced stimulation of nucleation. $\mathbf{c}$, Schematics illustrating formation of secondary domains in $\mathbf{b}$ when bias is applied only in primary locations. These data confirm that $x_{n+1}=q\left(x_{n}\right)$ is non-monotonic in the physical range of $r_{n}$ values, leading to chaotic behaviour.

Furthermore, these spatial interactions can enable experimental realization of cellular automata on the nanometre scale, potentially leading to new forms of information processing and contentretrieval memories. Although much more work is required to design a commercially attractive all-electronic computing device based on the domain interaction effect, the combination of SPM-control and dynamic (magnetoelectric readout ${ }^{38}$, topologically protected wall ${ }^{39}$ and conductive topological defects ${ }^{40}$ ) components could pave the way to these advances.

\section{Methods}

The sample was a $20-\mu \mathrm{m}$-thick plate of single-crystalline $\mathrm{LiNbO}_{3}$. A dimension 3100 (Veeco) scanning probe microscope equipped with a gas cell and a heating stage was used for measurements. The humidity of the ambient gas (nitrogen) was modified using a bubbler humidifier MG-101 (GE) and measured with a humidity meter 971 (Fluke). Custom-written Matlab codes were employed for modelling.

Received 26 March 2013; accepted 1 October 2013; published online 17 November 2013

\section{References}

1. Gleick, J. Chaos: Making a New Science (Penguin Books, 2008).

2. Strogatz, S. H. Nonlinear Dynamics And Chaos: With Applications To Physics, Biology, Chemistry, And Engineering (Westview, 2001).

3. Bunde, A. \& Havlin, S. Fractals and Disordered Systems (Springer, 2012).

4. Havlin, D. Diffusion and Reactions in Fractals and Disordered Systems (Cambridge Univ. Press, 2005).

5. Barabasi, A-L. \& Stanley, H. E. Fractal Concepts in Surface Growth (Cambridge Univ. Press, 1995).

6. Abarbanel, H. D. I., Brown, R., Sidorowich, J. J. \& Tsimring, L. S. The analysis of observed chaotic data in physical systems. Rev. Mod. Phys. 65, 1331-1392 (1993).

7. Ott, E., Grebogi, C. \& Yorke, J. A. Controlling chaos. Phys. Rev. Lett. 64, 1196-1199 (1990).

8. Garstecki, P., Fuerstman, M. J. \& Whitesides, G. M. Oscillations with uniquely long periods in a microfluidic bubble generator. Nature Phys. 1, 168-171 (2005).

9. Gruverman, A. \& Kholkin, A. Nanoscale ferroelectrics: Processing, characterization and future trends. Rep. Prog. Phys. 69, 2443-2474 (2006).

10. Kalinin, S. V., Morozovska, A. N., Chen, L. Q. \& Rodriguez, B. J. Local polarization dynamics in ferroelectric materials. Rep. Prog. Phys. 73, 056502 (2010).

11. Rodriguez, B. J. et al. Domain growth kinetics in lithium niobate single crystals studied by piezoresponse force microscopy. Appl. Phys. Lett. 86, 012906 (2005).
12. Woo, J. et al. Quantitative analysis of the bit size dependence on the pulse width and pulse voltage in ferroelectric memory devices using atomic force microscopy. J. Vac. Sci. Technol. B 19, 818-824 (2001).

13. Tybell, T., Paruch, P., Giamarchi, T. \& Triscone, J. M. Domain wall creep in epitaxial ferroelectric $\mathrm{Pb}\left(\mathrm{Zr}_{0.2} \mathrm{Ti}_{0.8}\right) \mathrm{O}_{3}$ thin films. Phys. Rev. Lett. 89, 097601 (2002).

14. Bonnell, D. A., Kalinin, S. V., Kholkin, A. L. \& Gruverman, A. Piezoresponse force microscopy: A window into electromechanical behavior at the nanoscale. MRS Bull. 34, 648-657 (2009).

15. Tanaka, K. et al. Scanning nonlinear dielectric microscopy nano-science and technology for next generation high density ferroelectric data storage. Jpn J. Appl. Phys. 47, 3311-3325 (2008).

16. Shen, J. et al. Study of asymmetric charge writing on $\mathrm{Pb}(\mathrm{Zr}, \mathrm{Ti}) \mathrm{O}_{3}$ thin films by Kelvin probe force microscopy. Appl. Surf. Sci. 252, 8018-8021 (2006).

17. Franke, K., Besold, J., Haessler, W. \& Seegebarth, C. Modification and detection of domains on ferroelectric PZT films by scanning force microscopy. Surf. Sci. 302, L283-L288 (1994).

18. Morozovska, A. N. et al. Piezoresponse force spectroscopy of ferroelectric-semiconductor materials. J. Appl. Phys. 102, 114108 (2007).

19. Tagantsev, A. K., Cross, L. E. \& Fousek, J. Domains in Ferroic Crystals and Thin Films (Springer, 2010).

20. Kalinin, S. V. \& Bonnell, D. A. Screening phenomena on oxide surfaces and its implications for local electrostatic and transport measurements. Nano Lett. 4, 555-560 (2004).

21. Kumar, A. et al. Probing surface and bulk electrochemical processes on the $\mathrm{LaAlO}_{3}-\mathrm{SrTiO}_{3}$ interface. ACS Nano 6, 3841-3852 (2012).

22. Watanabe, Y., Okano, M. \& Masuda, A. Surface conduction on insulating $\mathrm{BaTiO}_{3}$ crystal suggesting an intrinsic surface electron layer. Phys. Rev. Lett. 86, 332-335 (2001).

23. Fridkin, V. M. Ferroelectric Semiconductors (Springer, 1980).

24. Kalinin, S. V. \& Bonnell, D. A. Local potential and polarization screening on ferroelectric surfaces. Phys. Rev. B 63, 125411 (2001).

25. Kalinin, S. V., Johnson, C. Y. \& Bonnell, D. A. Domain polarity and temperature induced potential inversion on the $\mathrm{BaTiO}_{3}(100)$ surface. J. Appl. Phys. 91, 3816-3823 (2002).

26. Son, J. Y., Kyhm, K. \& Cho, J. H. Surface charge retention and enhanced polarization effect on ferroelectric thin films. Appl. Phys. Lett. 89, 092907 (2006).

27. Kim, Y., Hong, S., Kim, S. H. \& No, K. Surface potential of ferroelectric domain investigated by Kelvin force microscopy. J. Electroceram. 17, 185-188 (2006).

28. Rodriguez, B. J., Jesse, S., Baddorf, A. P., Kim, S. H. \& Kalinin, S. V. Controlling polarization dynamics in a liquid environment: From localized to macroscopic switching in ferroelectrics. Phys. Rev. Lett. 98, 247603 (2007).

29. Rodriguez, B. J., Jesse, S., Baddorf, A. P. \& Kalinin, S. V. High resolution electromechanical imaging of ferroelectric materials in a liquid environment by piezoresponse force microscopy. Phys. Rev. Lett. 96, 237602 (2006). 
30. Morozovska, A. N., Eliseev, E. A. \& Kalinin, S. V. The piezoresponse force microscopy of surface layers and thin films: Effective response and resolution function. J. Appl. Phys. 102, 074105 (2007).

31. Morozovska, A. N., Eliseev, E. A., Bravina, S. L. \& Kalinin, S. V. Resolution-function theory in piezoresponse force microscopy: Wall imaging, spectroscopy, and lateral resolution. Phys. Rev. B 75, 174109 (2007)

32. Kalinin, S. V. et al. Quantitative determination of tip parameters in piezoresponse force microscopy. Appl. Phys. Lett. 90, 212905 (2007).

33. Eliseev, E. A., Kalinin, S. V., Jesse, S., Bravina, S. L. \& Morozovska, A. N. Electromechanical detection in scanning probe microscopy: Tip models and materials contrast. J. Appl. Phys. 102, 014109 (2007).

34. Molotskii, M. I. \& Shvebelman, M. M. Dynamics of ferroelectric domain formation in an atomic force microscope. Phil. Mag. 85, 1637-1655 (2005).

35. Aravind, V. R. et al. Correlated polarization switching in the proximity of a 180 degrees domain wall. Phys. Rev. B 82, 024111 (2010).

36. Di Ventra, M. \& Pershin, Y. V. The parallel approach. Nature Phys. 9, 200-202 (2013).

37. Pershin, Y. V. \& Di Ventra, M. Memory effects in complex materials and nanoscale systems. Adv. Phys. 60, 145-227 (2011).

38. Spaldin, N. A. \& Fiebig, M. The renaissance of magnetoelectric multiferroics. Science 309, 391-392 (2005).

39. Wu, W. D., Horibe, Y., Lee, N., Cheong, S. W. \& Guest, J. R. Conduction of topologically protected charged ferroelectric domain walls. Phys. Rev. Lett. 108, 077203 (2012)

40. Seidel, J. et al. Conduction at domain walls in oxide multiferroics. Nature Mater 8, 229-234 (2009).

\section{Acknowledgements}

A part of this research (S.J., E.S., A.K., S.V.K.) was conducted at the Center for Nanophase Materials Sciences, which is sponsored at Oak Ridge National Laboratory by the Scientific User Facilities Division, Office of Basic Energy Sciences, US Department of Energy. A.V.I. and V.Y.S. acknowledge CNMS user proposal, RFBR (Grants 11-02-91066-CNRS-a, 13-02-01391-a, 13-02-96041-r-Ural-a), Ministry of Education and Science (Contract 14.513.12.0006). Y.V.P. was supported by National Science Foundation grant ECCS-1202383. The authors gratefully acknowledge Y. Wu (Tufts University) for posting the original version of the chaos analysis codes on the MathWorks website. A.N.M. and E.A.E. acknowledge the support through the bilateral SFFR-NSF project (US National Science Foundation under NSF-DMR-1210588 and State Fund of Fundamental State Fund of Fundamental Research of Ukraine, grant UU48/002). We gratefully acknowledge A. K. Tagantsev (EPFL) for valuable advice on the role of screening phenomena on ferroelectric phase stability, and B. Sumpter and S. Pennycook (ORNL) for illuminating discussions. S.V.K. and V.Y.S. would like to acknowledge many useful discussions with the late Y. D. Tretyakov (Moscow State University, Russia), who introduced them to the field of chaos and fractals in solid-state systems and inspired this work, and dedicate this paper to him in memoriam.

\section{Author contributions}

A.V.I. obtained and analysed the experimental data. S.V.K. proposed the concept and wrote (with A.N.M. and A.V.I.) the paper. E.S. and A.K. assisted in development of the experimental set-up and development of modelling codes. A.N.M. and E.A.E. developed theoretical analysis of the screening process and derived recursive formulae for domains in the chain. S.J. wrote the codes for simulating chaotic dynamics. Y.V.P. analysed the applications of the observed phenomena in information technology. S.V.K. and V.Y.S. directed the research.

\section{Additional information}

Supplementary information is available in the online version of the paper. Reprints and permissions information is available online at www.nature.com/reprints. Correspondence and requests for materials should be addressed to V.Y.S. or S.V.K.

\section{Competing financial interests}

The authors declare no competing financial interests. 